\title{
CÂNCER GÁSTRICO: FATORES DE RISCO EM CLIENTES ATENDIDOS NOS SERVIÇOS DE ATENÇÃO TERCIÁRIA EM UM MUNICÍPIO DO INTERIOR PAULISTA
}

Jesislei Bonolo do Amaral Teixeira ${ }^{1}$

Maria Suely Nogueira ${ }^{2}$

Teixeira JBA, Nogueira MS. Câncer gástrico: fatores de risco em clientes atendidos nos serviços de atenção terciária em um município do interior paulista. Rev Latino-am Enfermagem 2003 janeiro-fevereiro; 11(1):43-8.

Realizamos o presente estudo com clientes com diagnóstico de câncer gástrico e submetidos a cirurgia, com o objetivo de identificar a exposição aos fatores de risco para a doença. Foram sujeitos do estudo 24 clientes que concordaram em participar através da assinatura do termo de consentimento livre, respondendo a um instrumento semi-estruturado, elaborado segundo a perspectiva do Campo de Saúde. A análise dos dados possibilitou identificar que 50,1\% apontaram como fatores responsáveis dieta inadequada, tabagismo, etilismo e problemas psicológicos. Dentre o consumo de alimentos considerados de risco, encontramos tendência de maior consumo de enlatados, embutidos, defumados, churrasco e frituras, além de indicação de dietas com alto teor de sódio. Fumantes foram encontrados $66,7 \%$ ou que já fumaram e $70,8 \%$ indicaram consumo de bebida alcoólica, preferencialmente cerveja e destilados. Esses dados indicam a necessidade de implementação de programas educativos que visem o esclarecimento sobre os fatores de risco com vistas à promoção da saúde.

DESCRITORES: câncer gástrico, fatores de risco, promoção da saúde

\section{GASTRIC CANCER: RISK FACTORS IN CLIENTS ASSISTED IN THE TERTIARY CARE SERVICE AT A MUNICIPALITY IN THE INTERIOR OF SÃO PAULO STATE}

This study was developed with gastric cancer patients submitted to surgery with the purpose to identify their exposure to risk factors for the disease. Twenty-four clients who had agreed to participate by signing an informed consent term and responding a semi-structured instrument elaborated according to the Health Field perspective were the subjects in the study. The data analysis allowed to identify that $50.1 \%$ referred to inadequate diet, smoking, alcoholism and psychological problems as the responsible factors. With regard to the consumption of food considered to be harmful, there was a tendency to larger consumption of canned food, sausages, smoked and barbecued meat and fried food in addition to diets with high sodium content. $66.7 \%$ smoke or used to smoke and $70.8 \%$ indicated the consumption of alcoholic drinks, preferably beer or distilled types. These data show the need to implement educational programs with the purpose to inform about risk factors aiming at health promotion.

DESCRIPTORS: gastric cancer, risk factors, health promotion

\section{CÁNCER GÁSTRICO: FACTORES DE RIESGO EN CLIENTES ATENDIDOS EN LOS SERVICIOS DE ATENCIÓN TERCIÁRIA EN UNA MUNICIPALIDAD DEL INTERIOR DEL ESTAdO DE SÃO PAULO}

Realizamos el presente estudio con clientes con diagnóstico de cáncer gástrico sometidos a cirugía, con el objetivo de identificar la exposición a los factores de riesgo para la enfermedad. Fueron sujetos del estudio 24 clientes que concordaron en participar, firmando el término de consentimiento informado y respondiendo a un instrumento semiestructurado, elaborado según la perspectiva del Campo de Salud. El análisis de los datos hizo posible identificar que 50.1\% apuntaron como factores responsables la dieta inadecuada, el tabaquismo, el alcoholismo y los problemas psicológicos. Dentro del consumo de alimentos considerados de riesgo, encontramos tendencia hacia el mayor consumo de enlatados, embutidos, ahumados, churrasco y fritos, además de indicación de dietas con gran cantidad de sodio. 66.7\% fuman o ya fumaron y $70.8 \%$ indicaron el consumo de bebida alcohólica, de preferencia cerveza y destilados. Estos datos indican la necesidad de implementación de programas educativos que busquen aclarar sobre los factores de riesgos y con objetivo de promoción de la salud.

\section{DESCRIPTORES: cáncer gástrico, factores de riesgo, promoción de la salud}

\footnotetext{
${ }^{1}$ Professor do Curso de Enfermagem da Universidade de Uberaba, Mestre em Enfermagem pelo Programa de Pós Graduação Área Enfermagem Fundamental, e-mail: jesisleimjl@netscape.net; ${ }^{2}$ Professor Doutor. Escola de Enfermagem de Ribeirão Preto da Universidade de São Paulo, Centro Colaborador da OMS para o desenvolvimento da pesquisa em enfermagem
} 
INTRODUÇÃO

No Brasil, o câncer gástrico ainda representa importante causa de óbito e se coloca entre as cinco localizações primárias mais comuns de mortes e de casos novos de câncer, em ambos os sexos ${ }^{(1)}$. Para o país, estima-se que os números de óbitos e casos novos de câncer gástrico esperados para o ano 2001 sejam, respectivamente, 7090 e 15260, entre homens, e 3675 e 7070 , entre mulheres ${ }^{(2)}$.

O câncer, por ser doença crônico-degenerativa, apresenta evolução prolongada e progressiva, exceto se for interrompido em alguma de suas fases, em geral caracteriza-se por longo período de latência, fase assintomática prolongada, envolvimento de múltiplos fatores de risco, com destaque para os fatores ambientais ${ }^{(3)}$.

A ocorrência do câncer gástrico vem sendo associada à exposição de fatores intrínsecos decorrentes da constituição genética e a fatores extrínsecos, como o uso de dietas com altas concentrações de cloreto de sódio, nitratos e nitritos contidos em alimentos defumados e frituras $^{(4-5)}$.

Estudos epidemiológicos comprovam a relação entre nutrição e câncer de estômago, indicando que a associação entre fator protetor com dieta composta por legumes frescos e frutas é altamente consistente em numerosos estudos e sugere que a vitamina $\mathrm{C}$ e o caroteno diminuem o risco de câncer de estômago ${ }^{(6-7)}$.

O álcool é conhecido como fator de risco por lesar a mucosa gástrica. Estudos epidemiológicos sugerem que o álcool possui papel fundamental na carcinogênese, especialmente para cânceres de boca, faringe, laringe, esôfago e estômago, devido ao fato de ter efeito maior nos tecidos diretamente expostos durante o consumo e por agir sinergeticamente com o tabaco (fumo) ${ }^{(8-9)}$.

Há referências ${ }^{(10)}$ na literatura em que autores associam o surgimento do câncer a traumas específicos e a condições psicológicas como o luto, a ansiedade, desapontamentos, perda de apoio emocional dos pais, entre outras. Afirmam ainda que há estudos mostrando que estados emocionais podem afetar a transformação de células normais em células cancerosas.

Em decorrência do câncer gástrico também estar associado a fatores de risco, relacionados ao estilo de vida, que poderiam ser evitados, e considerando o papel educativo do enfermeiro com vistas à prevenção de doenças, buscamos investigar os hábitos de vida de pacientes com diagnóstico de câncer gástrico confirmado com o objetivo de: caracterizá-los quanto a crenças, hábitos alimentares e padrões de consumo relacionados ao estilo de vida.

O conhecimento produzido poderá beneficiar enfermeiros e os demais profissionais da área de saúde à medida que possibilita o fornecimento de informações para melhor atuação na prevenção e educação dos indivíduos, através da abordagem voltada para conscientização dos mesmos, alertando quanto à importância na mudança de hábitos considerados de risco para o câncer gástrico, bem como auxilio para detecção precoce da doença através de observação dos sinais e sintomas iniciais da neoplasia. E, a longo prazo, fornecer subsídios para elaboração de programas educativos voltados para aspectos preventivos e de atenção ao diagnóstico precoce.

\section{REFERENCIAL TEÓRICO}

Desenvolvemos o presente estudo com base no modelo conceitual denominado "Campo de Saúde", onde sustenta que a saúde é determinada por um conjunto de fatores agrupáveis em quatro divisões ou categorias: biologia humana, ambiente, estilo de vida e serviços de saúde ${ }^{(11-12)}$.

A categoria Biologia humana envolve todos os fatos que se manifestam como conseqüência da constituição orgânica do indivíduo, incluindo sua herança genética e seus processos de maturação; o ambiente agrupa os fatores externos ao organismo, em suas dimensões física e social, sobre os quais o indivíduo exerce pouco ou nenhum controle; o estilo de vida refere-se ao conjunto das decisões que o indivíduo toma a respeito da sua saúde, no que se refere, por exemplo, às suas atividades de lazer e alimentação, estando, portanto, parcialmente sob seu controle. Do ponto de vista da saúde inclui decisões e hábitos pessoais ruins, possibilitando, assim, os riscos autocriados. Quando esses riscos resultam em doenças ou mortes, o estilo de vida da vítima pode ser considerado como sendo responsável por essas ocorrências. A Organização dos serviços de saúde se refere à disponibilidade, quantidade e qualidade dos recursos destinados aos cuidados com a saúde ${ }^{(12)}$.

\section{METODOLOGIA}

Este estudo de caráter descritivo foi realizado entre clientes atendidos nos serviços de atenção terciária 
à saúde de Ribeirão Preto-SP. A população constituiu-se de clientes com diagnóstico de câncer gástrico confirmado, segundo os tipos de câncer gástrico incluídos nessa categoria conforme classificação CID (Código Internacional de Doenças), 10ª revisão, e que foram submetidos a cirurgia no período de outubro de 1998 a outubro de 1999. A amostra constituiu-se de 24 clientes atendidos em 4 hospitais de Ribeirão Preto, sendo 1 hospital Universitário, 2 hospitais de atenção terciária particular e que atendem convênios e pacientes particulares e 1 que atende pacientes de convênios e do SUS, cujos diagnósticos foram disponibilizados pelas equipes de gastrocirurgia dos referidos serviços.

Os dados foram obtidos pelos pesquisadores, após aprovação do projeto e instrumento de coleta de dados pelo Comitê de Ética em Pesquisa do Hospital das Clínicas da Faculdade de Medicina de Ribeirão Preto-USP, através de instrumento de coleta de dados, o qual foi elaborado com base no modelo conceitual de "Campo de Saúde"(12) e em pesquisas de outros autores ${ }^{(13-14)}$.

Buscando caracterizar o estilo de vida dos clientes identificamos a exposição aos fatores de risco para câncer gástrico como: uso de alimentos defumados, enlatados, frituras, uso excessivo de sal, tipo de conservação dos alimentos, uso de bebidas alcoólicas, tabagismo e fatores emocionais, além da freqüência de ingestão de verduras, legumes e frutas ricas em vitamina $\mathrm{C}$ e caroteno, de acordo com alimentos ricos em vitamina $\mathrm{C}^{(8)}$. A entrevista estruturada foi aplicada individualmente, considerando um período mínimo de três dias após a cirurgia ou até que o cliente se encontrasse em condições favoráveis para fornecer informações. Destacam-se os clientes que concordaram em participar do estudo após serem esclarecidos sobre os objetivos do mesmo e assinarem o termo de consentimento livre e esclarecido As respostas foram classificadas de forma sistemática, permitindo a análise dos dados de forma descritiva e percentual.

\section{RESULTADOS}

Com o objetivo de buscar informações em relação às crenças dos clientes acerca dos fatores de risco relacionados ao câncer gástrico, questionamos a quais fatores relacionavam a ocorrência da doença. Podemos identificar que $4(16,7 \%)$ clientes relacionaram sua doença ao uso de dieta inadequada, $4(16,7 \%)$ ao tabagismo e o etilismo, outros $4(16,7 \%)$ referiram problemas psicológicos de depressão, tristezas e decepções, perda de familiares, $2(8,3 \%)$ indicaram outros fatores como gastrite anterior e história familiar de câncer gástrico na família, enquanto $10(41,6 \%)$ não a relacionaram a fator algum.

Observamos que os clientes atribuíram o surgimento de sua doença a algum fator, mencionando fatores de risco relacionados à doença e a fatores psicológicos.

Considerando que a dieta alimentar constitui um dos itens que caracteriza o estilo de vida e que há fatores de risco para o câncer gástrico relacionados à alimentação, optamos por investigar o hábito alimentar dos clientes, segundo a ingestão de alimentos que são considerados de risco para tal neoplasia.

Tabela 1 - Distribuição dos alimentos considerados de risco para o câncer gástrico e freqüência de ingestão dos mesmos pelos 24 clientes com diagnóstico de câncer gástrico, Ribeirão Preto-SP, 1999

\begin{tabular}{|c|c|c|c|c|c|c|c|c|c|c|c|c|}
\hline \multirow[t]{2}{*}{ Alimentos } & \multicolumn{2}{|c|}{$\begin{array}{c}\text { Não faz } \\
\text { us o }\end{array}$} & \multicolumn{2}{|c|}{ Raramente } & \multicolumn{2}{|c|}{$\begin{array}{c}\text { Inferior a } 3 \\
\text { vezes / } \\
\text { sem ana }\end{array}$} & \multicolumn{2}{|c|}{$\begin{array}{c}\text { Superior a } 3 \\
\text { vezes } / \\
\text { semana }\end{array}$} & \multicolumn{2}{|c|}{ Diariamente } & \multicolumn{2}{|c|}{ Total } \\
\hline & $\mathbf{n}$ & $\%$ & $\mathbf{n}$ & $\%$ & $\mathbf{n}$ & $\%$ & $\mathbf{n}$ & $\%$ & $\mathbf{n}$ & $\%$ & $\mathbf{n}$ & $\%$ \\
\hline Enlatados & 5 & $\begin{array}{l}28,3 \\
(7,5)\end{array}$ & 10 & $\begin{array}{c}41,7 \\
(12,0)\end{array}$ & 6 & $\begin{array}{c}25,0 \\
(11,1)\end{array}$ & 1 & $\begin{array}{c}4,2 \\
(6,2)\end{array}$ & 2 & $\begin{array}{c}8,3 \\
(10,4)\end{array}$ & 24 & 100 \\
\hline Conservas & 8 & $\begin{array}{l}33,3 \\
(12)\end{array}$ & 9 & $\begin{array}{c}37,5 \\
(10,7)\end{array}$ & 5 & $\begin{array}{l}20,8 \\
(9,3)\end{array}$ & 1 & $\begin{array}{c}4,2 \\
(6,2)\end{array}$ & 1 & $\begin{array}{c}4,2 \\
(5,3)\end{array}$ & 24 & 100 \\
\hline Salsicha & 7 & $\begin{array}{c}29,1 \\
(10,4)\end{array}$ & 7 & $\begin{array}{l}29,2 \\
(8,3)\end{array}$ & 7 & $\begin{array}{c}29,2 \\
(12,9)\end{array}$ & 2 & $\begin{array}{c}8,3 \\
(12,5)\end{array}$ & 1 & $\begin{array}{c}4,2 \\
(5,3)\end{array}$ & 24 & 100 \\
\hline $\begin{array}{l}\text { Presunto/ } \\
\text { mortadela }\end{array}$ & 6 & $\begin{array}{l}25,0 \\
(9,0)\end{array}$ & 7 & $\begin{array}{l}29,2 \\
(8,3)\end{array}$ & 7 & $\begin{array}{c}29,2 \\
(12,9)\end{array}$ & 2 & $\begin{array}{c}8,3 \\
(12,5)\end{array}$ & 2 & $\begin{array}{c}8,3 \\
(10,5)\end{array}$ & 24 & 100 \\
\hline Defumados & 11 & $\begin{array}{c}45,8 \\
(16,4)\end{array}$ & 8 & $\begin{array}{l}33,3 \\
(9,5)\end{array}$ & 2 & $\begin{array}{c}4,2 \\
(3,7)\end{array}$ & 2 & $\begin{array}{c}8,3 \\
(12,5)\end{array}$ & 1 & $\begin{array}{c}4,2 \\
(5,3)\end{array}$ & 24 & 100 \\
\hline Carne seca & 5 & $\begin{array}{l}20,8 \\
(7,5)\end{array}$ & 11 & $\begin{array}{c}45,9 \\
(13,1)\end{array}$ & 6 & $\begin{array}{c}25,0 \\
(11,1)\end{array}$ & 2 & $\begin{array}{c}8,3 \\
(12,5)\end{array}$ & - & - & 24 & 100 \\
\hline Peixe salgado & 11 & $\begin{array}{c}45,8 \\
(16,4)\end{array}$ & 7 & $\begin{array}{l}29,2 \\
(8,3)\end{array}$ & 4 & $\begin{array}{l}16,7 \\
(7,4)\end{array}$ & 1 & $\begin{array}{c}4,2 \\
(6,2)\end{array}$ & 1 & $\begin{array}{c}4,2 \\
(5,3)\end{array}$ & 24 & 100 \\
\hline Peixe em conserva & 6 & $\begin{array}{l}25,0 \\
(9,0)\end{array}$ & 10 & $\begin{array}{c}41,7 \\
(12,0)\end{array}$ & 6 & $\begin{array}{c}25,0 \\
(11,1)\end{array}$ & 1 & $\begin{array}{c}4,2 \\
(6,2)\end{array}$ & 1 & $\begin{array}{c}4,2 \\
(5,3)\end{array}$ & 24 & 100 \\
\hline Churrasco & 4 & $\begin{array}{l}16,7 \\
(5,9)\end{array}$ & 9 & $\begin{array}{c}37,5 \\
(10,7)\end{array}$ & 8 & $\begin{array}{c}33,3 \\
(14,8)\end{array}$ & 3 & $\begin{array}{c}12,5 \\
(18,9)\end{array}$ & - & - & 24 & 100 \\
\hline Alimentos fritos & 4 & $\begin{array}{l}16,7 \\
(5,9)\end{array}$ & 6 & $\begin{array}{l}25,0 \\
(7,1)\end{array}$ & 3 & $\begin{array}{l}12,5 \\
(5,6)\end{array}$ & 1 & $\begin{array}{c}4,2 \\
(6,2)\end{array}$ & 10 & $\begin{array}{c}41,6 \\
(52,6)\end{array}$ & 24 & 100 \\
\hline Total & 67 & $(100)$ & 84 & $(100)$ & 54 & 100 & 16 & 100 & 19 & 100 & & \\
\hline
\end{tabular}


Pelos dados da Tabela 1, observamos que, em relação à ingesta de alimentos de risco para o câncer gástrico e considerando o não uso, o uso raro ou o uso inferior a três vezes por semana, a freqüência de citações foi de 205, contra 34 citações para a indicação de uso diário ou superior a três vezes por semana.

Dentre os alimentos indicados de uso diário e/ou de uso superior a três vezes na semana, verificamos tendência maior no consumo de enlatados, mortadela e presunto, salsicha, defumados, churrasco e alimentos fritos.

Estudos epidemiológicos ${ }^{(8)}$ indicaram aumento nos riscos de cânceres de estômago e esôfago com ingesta freqüente de alimentos fritos e defumados.

De acordo com o levantamento bibliográfico, encontramos que, assim como existem fatores dietéticos de risco para a doença, há também aqueles que são protetores. Dessa forma, procuramos identificar se esses últimos faziam parte do hábito alimentar dos entrevistados.

Buscamos identificar a freqüência de ingestão de verduras, legumes e frutas ricas em vitamina $\mathrm{C}$ e caroteno, de acordo com tabela dos alimentos ricos em vitamina $C$ e caroteno ${ }^{(8)}$ e observamos, através das respostas, a relação ao consumo de alimentos protetores contra o câncer gástrico, no qual a maioria dos clientes de nosso estudo se enquadrariam na categoria "não faz uso", tanto para frutas, como para legumes e verduras; ao lado disso o uso diário/ou superior a três vezes/semana foram as categorias menos citadas, tanto para legumes e verduras como para as frutas.

E estudo epidemiológico ${ }^{(6)}$ comprova a relação entre nutrição e câncer de estômago, indicando que a associação entre fator protetor com dieta composta por legumes frescos e frutas é altamente consistente em numerosas investigações, sugerindo que a vitamina $\mathrm{C}$ e o caroteno diminuem o risco de câncer de estômago.

Considerando que a ingesta de dietas com alto teor de sódio constitui fator de risco para o desenvolvimento de câncer gástrico, questionamos os clientes sobre a quantidade diária aproximada de sal. Para chegarmos a um valor aproximado, fizemos a seguinte pergunta: "Por quanto tempo tem duração um quilo de sal, considerando o número de pessoas que compartilham da alimentação preparada com o mesmo, na sua residência?". Dessa forma, fizemos uma média entre a quantidade de sal usada e o número de pessoas que realizavam refeições na casa, chegando a uma quantidade aproximada de sal que cada um ingeria por dia.

É importante esclarecer que não foi nosso objetivo um aprofundamento nesse tema, uma vez que sua complexidade exige um estudo à parte. No entanto, chegamos a um valor indicativo de que o consumo diário de sal, por pessoa, variou de 3,5 a 16,5g, indicando que 0 usavam em excesso*.

Dietas com altas concentrações de cloreto de sódio podem lesar a mucosa gástrica, tornando-a mais susceptível à ação de carcinógenos químicos, além da possibilidade de gastrite crônica ${ }^{(15)}$.

Quando questionados sobre a forma de conservação dos alimentos, todos indicaram usar a geladeira, exceto 1 deles que referiu ter tal eletrodoméstico há apenas quatro anos e que anteriormente a conservação se dava através do cozimento e manutenção em gordura animal e temperatura ambiente. Sobre esse aspecto parece que, para esse grupo de clientes, a conservação dos alimentos é adequada, não se constituindo em fator de risco.

O tabagismo foi outro fator de risco para o câncer gástrico investigado. Foi observado que 16 (66,7\%) clientes fumaram ou ainda estavam fumando. Esses últimos indicaram ter o hábito há pelo menos por um período em média de 35 a 50 anos; quanto aos ex-fumantes, referiram ter fumado por um período de 20 a 40 anos.

Em estudo realizado entre clientes com diagnóstico de neoplasia gástrica e de esôfago, concluiuse que há um risco aumentado em $2,4 \%$ das chances para o câncer gástrico, entre os fumantes. Há, no entanto, pequena redução neste risco, após um período de trinta anos de interrupção do hábito de fumar, sendo que há o aumento de intensidade conforme o tempo de uso do fumo. É ressaltado que o hábito de fumar responde por aproximadamente $40 \%$ dos casos de neoplasia de cárdia gástrica $^{(16)}$.

Em relação ao consumo de bebida alcoólica, 17 $(70,8 \%)$ clientes indicaram o seu uso, contra $7(29,1 \%)$ que negaram.

Observamos que assim como o tabagismo, o hábito de ingerir bebidas alcoólicas está presente na maioria dos clientes de nosso estudo.

O álcool parece ter efeito maior nos tecidos

\footnotetext{
${ }^{*}$ A ingesta de dieta com 6 a 8 gramas de sal/dia é uma quantidade limite para proteção do câncer gástrico ${ }^{(5)}$
} 
diretamente expostos durante seu consumo e age sinergeticamente com tabaco/fumo. A referida substância poderia agir indiretamente deprimindo a resposta imunológica, alterando o metabolismo das células epiteliais, aumentando a absorção de carcinógenos ou elevando a susceptibilidade ao mesmo ${ }^{(9)}$.

Os dados mostram que os clientes de nosso estudo têm tendência em consumir mais cervejas e destilados. Em relação ao consumo de cerveja, a maioria, ou seja, 6 (35,3\%), indicou fazer uso de uma a duas cervejas/dia, enquanto que para destilados 5 (29,4\%) referiram usar quantidade superior a duas doses diárias, sendo o aguardente a bebida mais freqüentemente citada. Podemos verificar o uso crônico de bebida alcoólica, indicada por um período que variou de 10 a 50 anos, sendo mais freqüente a indicação de tempo superior a 40 anos.

Durante a coleta de dados, alguns clientes referiram que nos finais de semana chegavam a ingerir maior quantidade de bebida alcoólica, correspondendo, muitas vezes, a uma garrafa de aguardente e várias cervejas.

Observamos, portanto, um número significativo de clientes etilistas e com ingesta de altas doses de bebida alcoólica diária, considerando que é considerada ${ }^{(17)}$ dose excessiva de bebida alcoólica o correspondente a $39 \mathrm{ml}$ de álcool/dia, eqüivalendo a $60 \mathrm{ml}$ de vinho, duas doses de licor, rum, uísque e aguardente ou duas garrafas de cerveja.

Quando buscamos informações de algum fator estressante por eles vivido, destacando o seu aparecimento anteriormente à doença, destarte a ocorrência de algum fato que pudesse ter trazido tristeza, contrariedade ou mesmo dificuldades, 16 (66,6\%) indicaram que haviam passado por fatores estressantes, como a perda de familiares, esposo ou esposa, netos, irmãos, sogro ou sogra, e referiram que, além do sofrimento da perda, surgiram dificuldades relacionadas a problemas financeiros, sobrecarga de responsabilidade e solidão, bem como outros problemas relacionados aos filhos e esposo, diminuição da renda familiar motivada por desemprego, separação, afora problemas no trabalho. Indicaram a ocorrência desses problemas por um período que variou de 4 meses a 13 anos, antes do surgimento dos primeiros sintomas indicativos de problemas gástricos, como azia, má digestão.

Observamos número considerável de clientes que referiram dificuldades anteriores ao surgimento do problema gástrico, sendo que durante a entrevista sentiam necessidade de contar suas experiências de vida, que, no geral, sempre estiveram marcadas por privações, dificuldades financeiras, ou mesmo situações causadoras de estresse, conflitos e tristezas, o que, talvez, tenha contribuído para afetar hábitos de vida e o estado de humor.

O ambiente psicológico negativo com nível social baixo, estresse e o modo com que as pessoas enfrentam a situação estressante podem ser desencadeadores de uma variedade de doenças ${ }^{(4)}$.

\section{CONCLUSÕES E CONSIDERAÇÕES FINAIS}

Esta pesquisa permitiu verificar que os clientes com diagnóstico de câncer gástrico atribuíram o surgimento da doença a algum fator, mencionando fatores de risco para a mesma; observamos que, mesmo com maior número de citações de uso raro ou o uso inferior a três vezes por semana de alimentos considerados de risco, a ingesta de alimentos considerados protetores também não fazia parte dos hábitos alimentares de nossa amostra. No preparo das refeições, faziam uso de sal em excesso e todos conservavam os alimentos na geladeira; em sua maioria já fumaram ou ainda estavam fumando; referiram ingerir bebidas alcoólicas por longo período de tempo e em altas doses diárias, aumentando a quantidade e intensidade quando do surgimento de problemas familiares, financeiros e psicológicos. Observamos um número considerável de clientes que referiram dificuldades anteriores ao problema gástrico.

A caracterização feita tem implicações práticas para a identificação dos grupos e fatores de risco para desenvolvimento do câncer gástrico e podem ser utilizadas para conscientizar os profissionais de saúde sobre a implementação de programas de prevenção e controle da doença, através da educação e conscientização da população acerca dos fatores de risco passíveis de modificação. 


\section{REFERÊNCIAS BIBLIOGRÁFICAS}

1. Banco Mundial. Brasil novo desafio à saúde do adulto. Washington; 1991.

2. Ministério da Saúde (BR). Instituto Nacional de Câncer. Coordenação Nacional de controle de tabagismo-CONTAPP. Falando sobre Câncer e seus Fatores de Risco. Rio de Janeiro, [serie online] 1996. [citado 2001 julho 10]; Disponível em: URL:http://http://www.inca.org.br/epidemiologia/ estimativa2001/introducao.html

3. Ministério da Saúde (BR). Secretaria Nacional de Assistência á Saúde. Instituto Nacional do Câncer. Coordenação de Programas de Controle do Câncer. ProOnco. Ações de enfermagem para controle do câncer. Rio de Janeiro: Pro-Onco; 1995.

4. Carneiro MRG, Pinto LFR, Paumgartten FJR. Fatores de risco ambientais para o câncer gástrico: a visão toxicologista. Cad Saúde Publica 1997; 13 (supl 1):27-38.

5. Abreu E. A prevenção primária e detecção do câncer de estômago. Cad Saúde Publica 1997; 13 (Supl 1):105-8.

6. Kono S, Hirohata T. Nutrition and Stomach cancer. Cancer Causes Controle 1996; 7(1):41-55.

7. Latorre MRDO. A mortalidade por câncer de estômago no Brasil: análise do período de 1977 a 1989. Cad Saúde Pública 1997; 13(Supl 1):67-78.

8. Mahan LKK. Alimentos, Nutrição e Dietoterapia. $8^{\mathfrak{a}}$ ed. São Paulo: Roco; 1994.

9. Rogers EC, Canner MW. Alcohol and cancer. Adv Exp Med Biol 1986; 206-473.

10. Kovács MJ. Morte e Desenvolvimento Humano. São Paulo: Casa do Psicólogo; 1992.

11. Dever GEA. A epidemiologia na administração dos serviços de saúde. São Paulo: Pioneira; 1988.

12. Lalonde, M. A new perspective on the health of Canadians: a working document. Otawa: Minister of National Health and Welfare; 1974.

13. Colombo RCR. Estilo de vida e fatores de risco de clientes com primeiro episódio de infarto agudo do miocárdio. [Dissertação]. Ribeirão Preto (SP): Escola de Enfermagem de Ribeirão Preto/USP; 1995.

14. Dantas RAS. Perfil de clientes com infarto agudo na perspectiva do modelo de "Campo de Saúde". [Dissertação]. Ribeirão Preto (SP): Escola de Enfermagem de Ribeirão Preto/ USP; 1996.

15. Britto AV. Câncer de estômago: fatores de risco. Cad Saúde Pública 1997; 13 (supl 1):7-13.

16. Gamman MD. Adenocarcinomas of the esophagus and gastric cardia and conditions tabaco, alcohol and socioeconomic factors. Nath Câncer Inst 1997 Sep; 89(17):1277-84.

17. International Lipid linformation Bureau (ILIB) Latino América. Recomendations de ILIB para el diagnóstico de las dislipidemias en Latinoamerica. Cardiovasc Risk Factors 1994; 3(1):10-27. 